Review Article

\title{
The Effects of Sesame Consumption on Glycemic Control in Adults: A Systematic Review and Meta-Analysis of Randomized Clinical Trial
}

\author{
Alireza Yargholi, ${ }^{1}$ Mohammad Hasan Najafi, ${ }^{2}$ Mohammad Ali Zareian $\mathbb{D}^{1},{ }^{1}$ Jessie Hawkins, ${ }^{3}$ \\ Laila Shirbeigi, ${ }^{1}$ and Mohammad Hossein Ayati ${ }^{1}{ }^{1}$ \\ ${ }^{1}$ Department of Traditional Medicine, School of Persian Medicine, Tehran University of Medical Sciences (TUMS), Tehran, Iran \\ ${ }^{2}$ Department of Traditional Medicine, Faculty of Iranian Traditional Medicine, Shahid Sadoughi University of Medical Sciences, \\ Ardakan, Yazd, Iran \\ ${ }^{3}$ Integrative Health, Franklin School of Integrative Health Sciences, Franklin, Tennessee, USA
}

Correspondence should be addressed to Mohammad Hossein Ayati; ayatimd@gmail.com

Received 9 May 2021; Revised 10 July 2021; Accepted 13 August 2021; Published 18 October 2021

Academic Editor: Vahidreza Ostadmohammadi

Copyright (c) 2021 Alireza Yargholi et al. This is an open access article distributed under the Creative Commons Attribution License, which permits unrestricted use, distribution, and reproduction in any medium, provided the original work is properly cited.

\begin{abstract}
Objectives. In recent years, diabetes has become a global health problem that creates a tremendous economic burden for many countries. Clinical trials evaluating the hypoglycemic effects of sesame consumption have produced conflicting results. This systematic review and meta-analysis was conducted to evaluate the effectiveness of sesame as a popular natural herb on glycemic indices in adults. Methods. The search for related articles in PubMed, Scopus, Google Scholar, and Cochrane library was conducted through May 2021. Results were reported as weighted mean differences (WMD) with 95\% confidence intervals (CI) using a random-effects model. Results. A total of 605 studies were identified through online searching, and a total of eight RCTs representing 382 participants were included in this study. The meta-analyses revealed that sesame consumption significantly decreases serum fasting blood sugar (FBS): (WMD: $-28.23 \mathrm{mg} / \mathrm{dl} ; 95 \%$ CI $(-39.16,-17.13), I^{2}=97.6 \%$; 95\% CI (96, 98)), and hemoglobin A1c (HbA1c): (WMD: $-1.00 \%$; 95\% CI $(-1.11,-0.88), I^{2}=0 \%$; 95\% CI $(0,79)$ ) as compared to the control group. Conclusion. This study provides evidence of the hypoglycemic effects of sesame consumption, particularly in diabetic patients. Additional RCTs on sesame and its preparations should be conducted in different populations to increase generalizability.
\end{abstract}

\section{Introduction}

Diabetes mellitus is one of the most common metabolic disorders, affecting over 420 million individuals worldwide [1]. This condition was responsible for 28.8 million deaths in 2016 [2]. Type 2 diabetes (T2D) is characterized by insulin resistance and is diagnosed by biochemical parameters such as high concentrations of serum blood sugar, insulin, and glycosylated hemoglobin ( $\mathrm{HbAlC})[3,4]$. Finding a practical approach to reduce the prevalence of diabetes is essential for global public and economic health. Herbal medicine, a component of complementary medicine (CAM), is commonly used to prevent and treat chronic conditions because of its lower costs and reduced side effects [5].
Sesame (Sesamum indicum L.) has been used as a traditional plant-based therapy worldwide, most commonly in Asian regions [6]. Sesame seed and its products (oil, flour, and dietary supplement) are good sources of lignan compounds (sesamin, sesamolin, sesamol, and episesamin) [7]. These lignans are responsible for most medicinal actions of sesame, including antioxidant activity [8], anti-inflammatory actions [9], and hypoglycemic [10] effects. Additionally, sesame contains high amounts of other beneficial components such as $\alpha$-tocopherol, polyunsaturated fatty acids (PUFAs), monounsaturated fatty acids (MUFAs), and fiber [11].

The hypoglycemic effects of sesame may be due to the action of its bioactive lignans which have been shown to 
improve insulin secretion from B cells of the pancreas [12]. Some animal studies have found that sesame consumption could reduce insulin resistance and enhance the glucose metabolism $[12,13]$. In human studies, sesame consumption has produced beneficial effects on serum glucose, HbA1C, and insulin concentrations in diabetic patients [14-16]. However, to the best of our knowledge, there has not been a systematic review and meta-analysis examining the effects of sesame consumption on glycemic control. The purpose of this study was to systematically assess all randomized clinical trials (RCTs) performed on the impact of sesame and sesame-based products on glycemic control in adults and conduct a meta-analysis to summarize our findings.

\section{Materials and Methods}

2.1. Search Strategy. We used the Preferred Reporting Items for Systematic Reviews and Meta-Analyses (PRISMA) guidelines for this study [17] (Supplementary Table 1).

PubMed/Medline, Cochrane Library, Google Scholar, and Scopus were searched to locate relevant RCTs published through May 2021. The search strategy was prepared using medical subject headings ( $\mathrm{MeSH}$ ) and dependent keywords as follows: (("Sesame" OR "Sesame Oil" OR "Sesame Seed" OR "Sesamum”) AND ("Blood Glucose” OR "Insulin” OR "Glycated Hemoglobin A" OR "Insulin Resistance" OR "Diabetes Mellitus" OR "Glucose" OR "Blood Glucose” OR "Insulin Resistance" OR “diabetes" OR T2DM OR "hyperglycemia” OR "hyperglycemia” OR "HbA1c" OR “insulin sensitivity" OR "HOMA" OR "glucose homeostasis "OR "insulin secretion" OR "beta-cell function" OR "glycemic control" OR "glucose tolerance" OR "glucose metabolism")). No restrictions were used when searching the databases. To ensure all related articles were identified, we checked the reference list of all qualified studies and related reviews.

2.2. Eligibility Criteria. Two investigators independently browsed the title and abstract of every article and included them if they met the inclusion criteria. To be included, the studies had to be RCTs (parallel or crossover) examining the effect of sesame seed and its preparations (oil/flour/supplement) on glycemic indices such as serum fasting blood sugar (FBS), hemoglobin A1C (HbA1C), insulin concentrations, insulin resistance (HOMA-IR: homeostasis model assessment of insulin resistance), and insulin sensitivity (QUICKI: quantitative insulin sensitivity check index).

Studies that used other compounds in addition to sesame only in the intervention group, studies without a control group, and studies with the same populations were excluded.

2.3. Data Extraction. Two researchers independently extracted the following data from every eligible article: author's name, publication year, country, sesame source and quantity, duration of intervention, participant's information (health condition, age, gender, and sample size), study type, and glycemic indices values (mean and standard deviation) before and after the intervention. Discrepancies were solved by consulting with the principal investigator.
2.4. Risk of Bias Assessment. Risk of bias was evaluated by two researchers independently using the Jadad checklist [18]. This scale contains three sections: randomization, blinding, and description of dropouts. Studies with score of $\geq 3$ were considered to be high quality.

2.5. Quality of Evidence. Quality of the evidence was evaluated using the Grades of Recommendation, Assessment, Development, and Evaluation (GRADE) tool [19]. This tool evaluates within-study risk of bias, inconsistency of results, indirectness of evidence, imprecision, and risk of publication bias. Studies were classified as high, moderate, low, or very low quality.

2.6. Statistical Method. Effect sizes were reported as weighted mean differences (WMD) and 95\% confidence intervals (CI) and were obtained by combining effect sizes of mean (SD) changes of glycemic control biomarkers using random-effects models. Due to the small number of the studies in these analyses, the Hartung-Knapp adjustment was also used [20]. We calculated mean change and standard deviation using following formula in studies where those values were not reported: mean change $=$ final values-baseline values; $\mathrm{SD}=$ square root ( $\mathrm{SD}$ baseline $) 2+(\mathrm{SD}$ final) $2-(2 \mathrm{R} \times \mathrm{SD}$ baseline $\times \mathrm{SD}$ final $))[21]$. A correlation coefficient equal to 0.8 was considered the $R$ value when calculating SD change [21]. Between study heterogeneity was assessed using the $I^{2}$ index and its confidence interval [22]. Low, moderate, and high heterogeneities were ascribed to $I^{2}$ values of $25-50 \%, 50-75 \%$, and $>75 \%$, respectively [23]. Predetermined subgroup analyses were conducted on factors including type of sesame product (sesame seed, sesame oil, and sesamin supplement), sample size, duration of intervention, and quality of studies. Sensitivity analyses were performed to determine the influence of each individual study on the overall effect size. Potential publication bias was evaluated using Egger's weighted regression test, as well as visual inspection of funnel plots. All statistical procedures were performed using STATA (Version 12.0, Stata Corp., College Station, TX). Statistical significance was defined as $P<0.05$.

\section{Results}

3.1. Search Results. A total of 605 articles were entered into Endnote for screening, as shown in Figure 1. After reviewing the titles, abstracts, and subsequently full-texts of remained articles and removing duplicates, eight RCTs [14-16, 24-28] were included in this systematic review.

General characteristics of the included studies are reported in Table 1 . These studies were published between 2006 and 2020 and were conducted in Iran [14, 25, 26, 28], India [16, 27], Brazil [24], and Pakistan [15]. The age of the participants and the duration of intervention were 18-70 years and 6-12 weeks, respectively. A total of 262 participants took part in sesame intervention, with 255 in control groups. Only one article was conducted on women [24], and all other trials included males and females [14-16, 25-28]. 


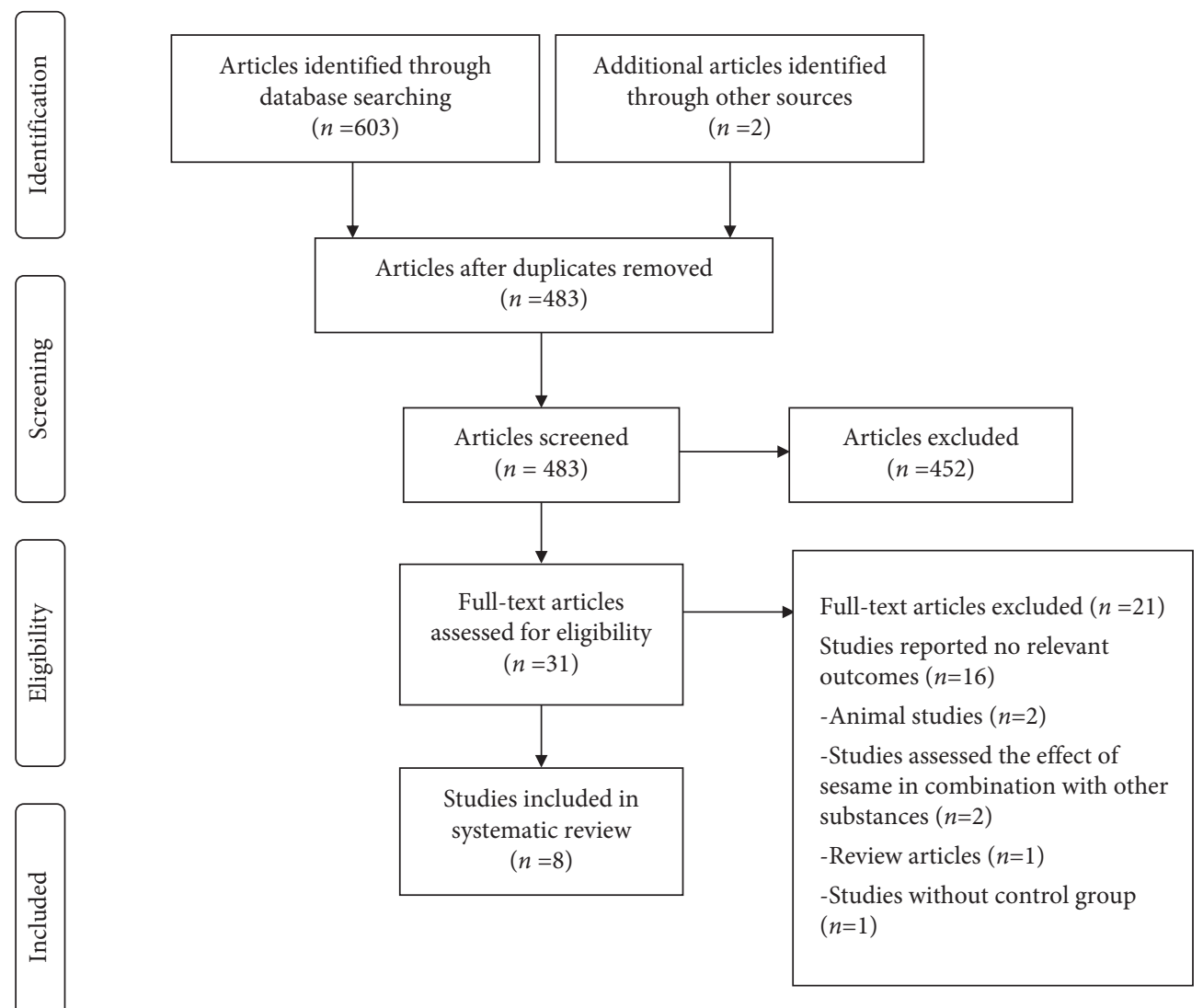

Figure 1: Flow diagram of the included and excluded studies.

Sesame was evaluated as sesame oil [15, 16, 26-28], sesame seed $[24,25]$, and sesamin supplement [14]. Six RCTs had a parallel design [14-16, 24, 25, 28], while two studies had a crossover design $[26,27]$. Based on Jadad scale evaluations, four studies were considered to be high-quality $[14,25,26,28]$ (Table 2).

\subsection{Meta-Analysis}

3.2.1. The Effects of Sesame Intake on FBS. After pooling seven studies [14-16, 24, 25, 27, 28] evaluating the impact of sesame on FBS by the random effect model, we found that sesame consumption significantly reduces serum FBS levels as compared to a control group (WMD: $-28.23 \mathrm{mg} / \mathrm{dl} ; 95 \%$ CI $(-39.16,-17.13), I^{2}=97.6 \%$; 95\% CI $(96,98)$ ) (Figure 2). The results in Hartung-Knapp adjustment analysis are as follows: WMD $28.01 \mathrm{mg} / \mathrm{dl}$; 95\% CI (2.31, 53.70), $I^{2}=97.5 \%$. When evaluating heterogeneity, sesame preparation, duration of treatment, and sample size were identified as the sources (Table 3).

3.2.2. The Effect of Sesame Intake on $H b A 1 C$. Five studies $[14-16,24,27]$ that examined the effect of sesame on serum $\mathrm{HbA1C}$ levels were used in the meta-analysis. The result of the random effect model was WMD: $-1.00 \%$; 95\% CI ( -1.11 , $-0.88), I^{2}=0 \%$; $95 \%$ CI $(0,79)$ (Figure 3), and HartungKnapp adjustment analysis was WMD 0.99\%; 95\% CI (0.82,
1.16), $I^{2}=0 \%$ in the serum $\mathrm{HbA}_{1} \mathrm{C}$ level. Subgroup analysis findings are given in Table 3.

3.2.3. The Effects of Sesame Intake on Other Glycemic Control Markers. Meta-analysis of three studies [14, 15, 28] on the effect of sesame usage on the serum insulin level failed to show any significant results (WMD: random effect model: $7.51 \mathrm{IU} / \mathrm{ml}$; 95\% CI $(-3.81,18.83), I^{2}=98.3 \% \%$; 95\% CI (97, 99), Hartung-Knapp adjustment analysis $7.52 \mathrm{IU} / \mathrm{ml} ; 95 \%$ CI $\left.(-26.85,11.81), I^{2}=98.3 \%\right)$.

3.3. Sensitivity Analysis. The sensitivity analysis found that none of the studies dramatically influenced the overall findings of FBS, HbA1C, and insulin level (Supplementary Figures 1-3).

3.4. Publication Bias. After evaluating Egger test findings and funnel plots, no evidence of publication bias was detected regarding FBS $(P=0.35)$, HbA1c $(P=0.22)$, and insulin $(P=0.98)$ (Supplementary Figures $4-6)$.

3.5. Quality of Evidence. The quality of the evidence was evaluated through GRADE. We found that studies conducted on the effects of sesame supplementation on serum FBS and HbAlc were of low quality and those on insulin were very low quality (Table 4 ). 


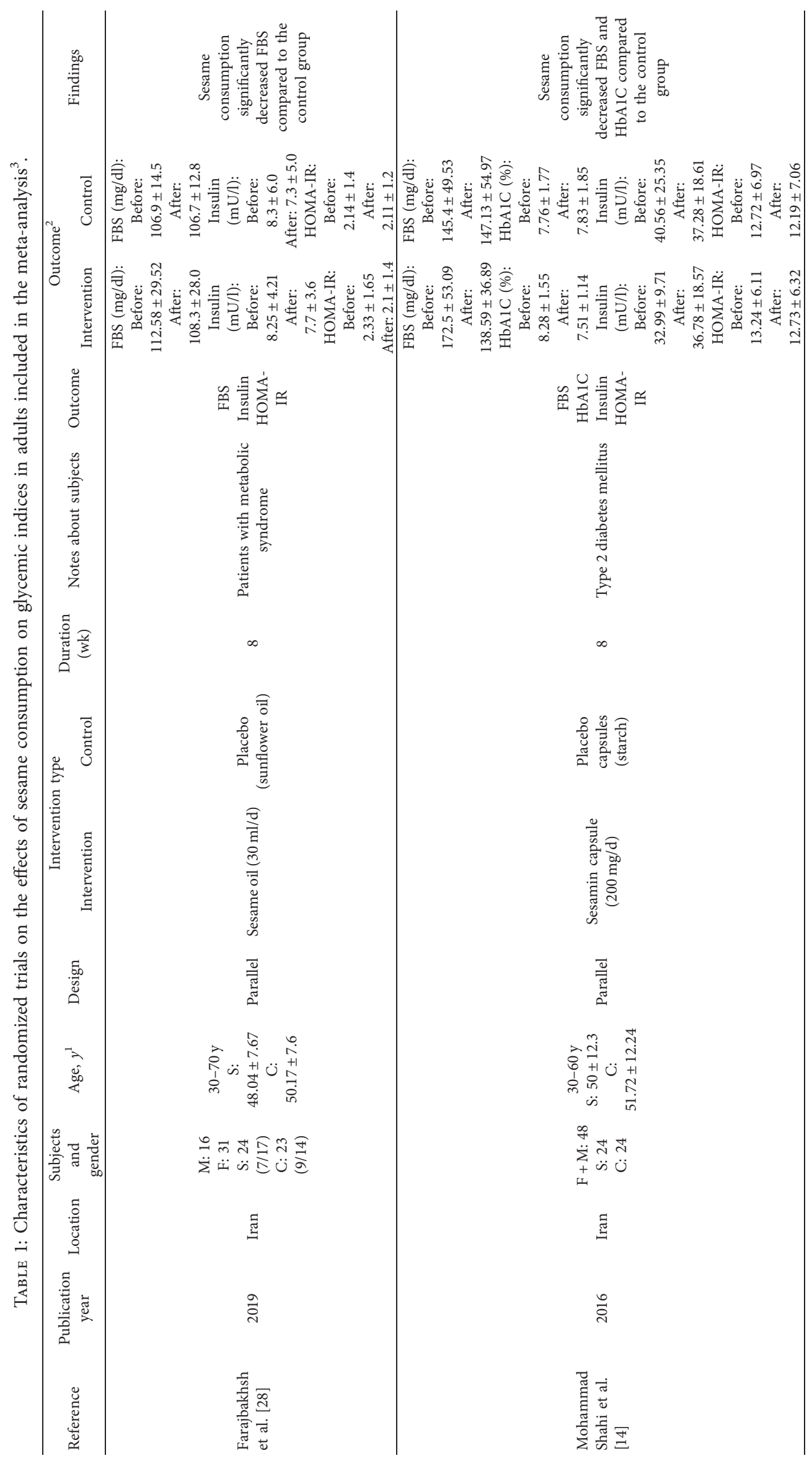




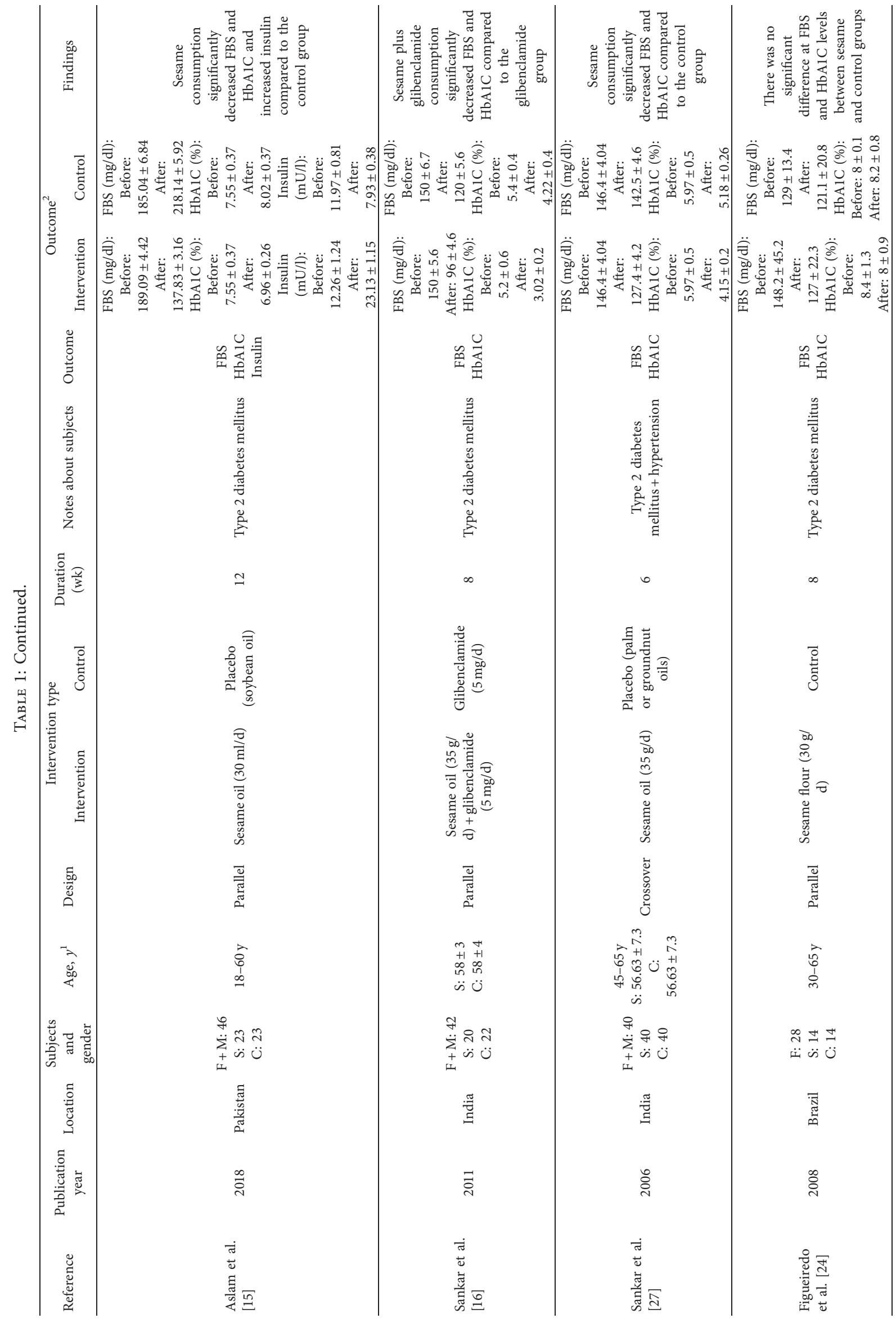




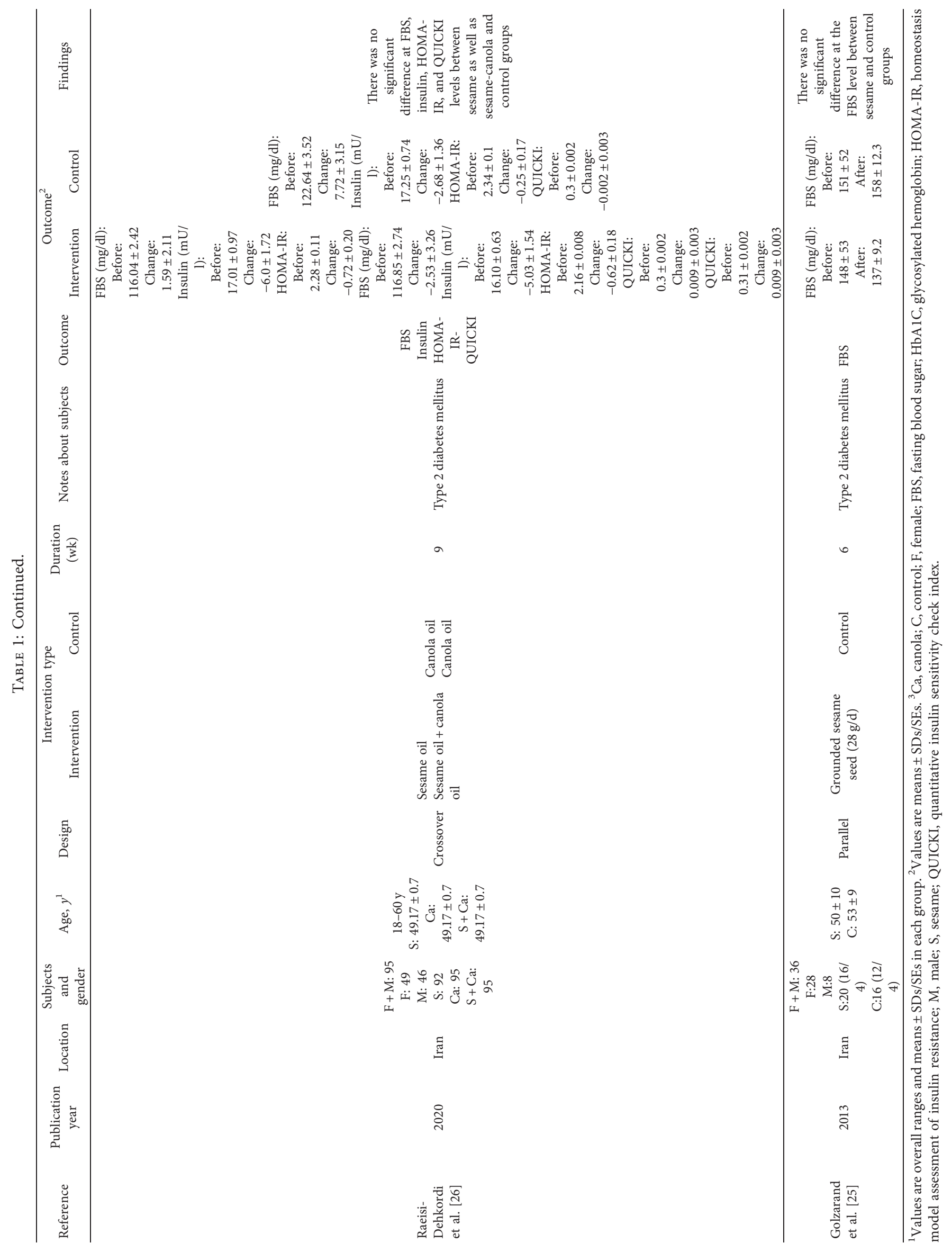


TABLE 2: Jadad risk of bias assessment for randomized controlled trials on the effect of sesame consumption on diabetic indices in adults.

\begin{tabular}{|c|c|c|c|c|c|c|}
\hline Reference & Randomization & $\begin{array}{l}\text { Method of } \\
\text { randomization }\end{array}$ & $\begin{array}{l}\text { Blinding of participants, } \\
\text { personnel, and outcome assessors }\end{array}$ & $\begin{array}{l}\text { Method of } \\
\text { blinding }\end{array}$ & $\begin{array}{l}\text { An account of } \\
\text { all patients }\end{array}$ & Total score \\
\hline Farajbakhsh et al. & $*$ & & $*$ & & $*$ & 3 \\
\hline Mohammad Shahi al. & * & & * & * & * & 4 \\
\hline Aslam et al. & * & & & & * & 2 \\
\hline Sankar et al. & $*$ & & & & $*$ & 2 \\
\hline Sankar et al & * & & & & $*$ & 2 \\
\hline Figueiredo et al & * & & & & * & 2 \\
\hline Raeisi-Dehkordi et al. & * & $*$ & $*$ & * & $*$ & 5 \\
\hline Golzarand et al. & $*$ & & $*$ & & $*$ & 3 \\
\hline
\end{tabular}

\begin{tabular}{|c|c|c|c|}
\hline $\begin{array}{l}\text { Study } \\
\text { ID }\end{array}$ & & WMD (95\% CI) & Weight (\%) \\
\hline Sankar et al. (2006) & $\bullet$ & $-15.10(-16.28,-13.92)$ & 17.94 \\
\hline Figueiredo et al (2008) & L. & $-13.30(-30.62,4.02)$ & 12.38 \\
\hline Sankar et al. (2011) & : & $-24.00(-26.26,-21.74)$ & 17.84 \\
\hline Golzarand et al (2013) & $\frac{1}{1} \cdot$ & $-18.00(-47.08,11.08)$ & 7.91 \\
\hline Shahi et al (2016) & $\rightarrow 1$ & $-35.64(-54.25,-17.03)$ & 11.82 \\
\hline Aslam et al (2018) & - & $-84.36(-94.00,-74.72)$ & 15.77 \\
\hline Farajbakhsh et al (2019) & it & $-4.00(-12.13,4.13)$ & 16.35 \\
\hline Overall $(I$-squared $=97.6 \%, p=0.000)$ & $\left\langle\begin{array}{l}1 \\
1\end{array}\right\rangle$ & $-28.23(-39.16,-17.30)$ & 100.00 \\
\hline Note: Weights are from random effects analysis & & & \\
\hline
\end{tabular}

FIGURE 2: Forest plot showing the effects of sesame consumption on circulating FBS in adults using the random effects model. Values are WMDs (95\% CIs) comparing changes in FBS over time between treatment and control groups. CI, confidence interval; FBS, fasting blood sugar; WMD, weighted mean difference.

TABLE 3: Pooled estimates of the effects of sesame supplementation on glycemic control biomarkers within different subgroups ${ }^{3}$.

\begin{tabular}{|c|c|c|c|c|c|c|c|c|c|c|}
\hline & \multicolumn{2}{|c|}{$\begin{array}{l}\text { Number of } \\
\text { trials }\end{array}$} & \multicolumn{2}{|c|}{ WMD (95\% CI) } & \multicolumn{2}{|c|}{$P$ value } & \multicolumn{2}{|c|}{$P$ heterogeneity } & \multicolumn{2}{|c|}{$I^{2}(\%)$} \\
\hline & FBS & HbAlc & FBS & $\mathrm{HbA1c}$ & FBS & $\mathrm{HbA1c}$ & FBS & $\mathrm{HbAlc}$ & FBS & $\mathrm{HbAlc}$ \\
\hline Total & 7 & 5 & $-28.22(-39.15,-17.29)$ & $-1.00(-1.11,-0.88)$ & $<0.001$ & $<0.001$ & $<0.001$ & 0.663 & 97.6 & 0 \\
\hline \multicolumn{11}{|c|}{ Type of intervention } \\
\hline Sesame capsule & 1 & 1 & $-35.64(-54.25,-17.02)$ & $-0.84(-1.43,-0.24)$ & $<0.001$ & 0.005 & - & - & - & - \\
\hline Sesame seed & 2 & 1 & $-14.53(-29.41,0.35)$ & $-0.60(-1.16,-0.03)$ & 0.056 & 0.036 & 0.786 & - & 0 & - \\
\hline Sesame oil & 4 & 3 & $-30.88(-44.33,-17.42)$ & $-1.02(-1.14,-0.89)$ & $<0.001$ & $<0.001$ & $<0.001$ & 0.971 & 98.8 & 0 \\
\hline \multicolumn{11}{|c|}{ Duration of treatment } \\
\hline$<8 \mathrm{wk}$ & 2 & 1 & $-15.10(-16.28,-13.92)$ & $-1.03(-1.18,-0.87)$ & $<0.001$ & $<0.001$ & 0.845 & - & 0 & - \\
\hline$=8 \mathrm{wk}$ & 4 & 3 & $-18.38(-31.58,-5.19)$ & $-0.93(-1.12,-0.74)$ & 0.006 & $<0.001$ & $<0.001$ & 0.407 & 87.8 & 0 \\
\hline$>8 \mathrm{wk}$ & 1 & 1 & $-84.36(-94.00,-74.72)$ & $-1.06(-1.78,-0.33)$ & $<0.001$ & 0.004 & - & - & - & - \\
\hline \multicolumn{11}{|l|}{ Sample size } \\
\hline$<40$ & 2 & 2 & $-14.53(-29.41,0.35)$ & $-0.90(-1.28,-0.52)$ & 0.056 & $<0.001$ & 0.786 & 0.147 & 0 & 52.3 \\
\hline$\geq 40$ & 5 & 3 & $-31.58(-43.98,-19.19)$ & $-0.98(-1.18,-0.78)$ & $<0.001$ & $<0.001$ & $<0.001$ & 0.866 & 98.4 & 0 \\
\hline \multicolumn{11}{|l|}{ Study quality } \\
\hline High & 3 & 1 & $-18.12(-40.39,4.14)$ & $-0.84(-1.43,-0.24)$ & 0.111 & 0.005 & 0.008 & - & 79.3 & - \\
\hline Low & 4 & 4 & $-34.32(-48.25,-20.40)$ & $-1.00(-1.12,-0.88)$ & $<0.001$ & $<0.001$ & $<0.001$ & 0.547 & 98.7 & 0 \\
\hline
\end{tabular}

CI, confidence interval; FBS, fasting blood sugar; HbAlc, hemoglobin Alc; WMD, weighted mean difference. 


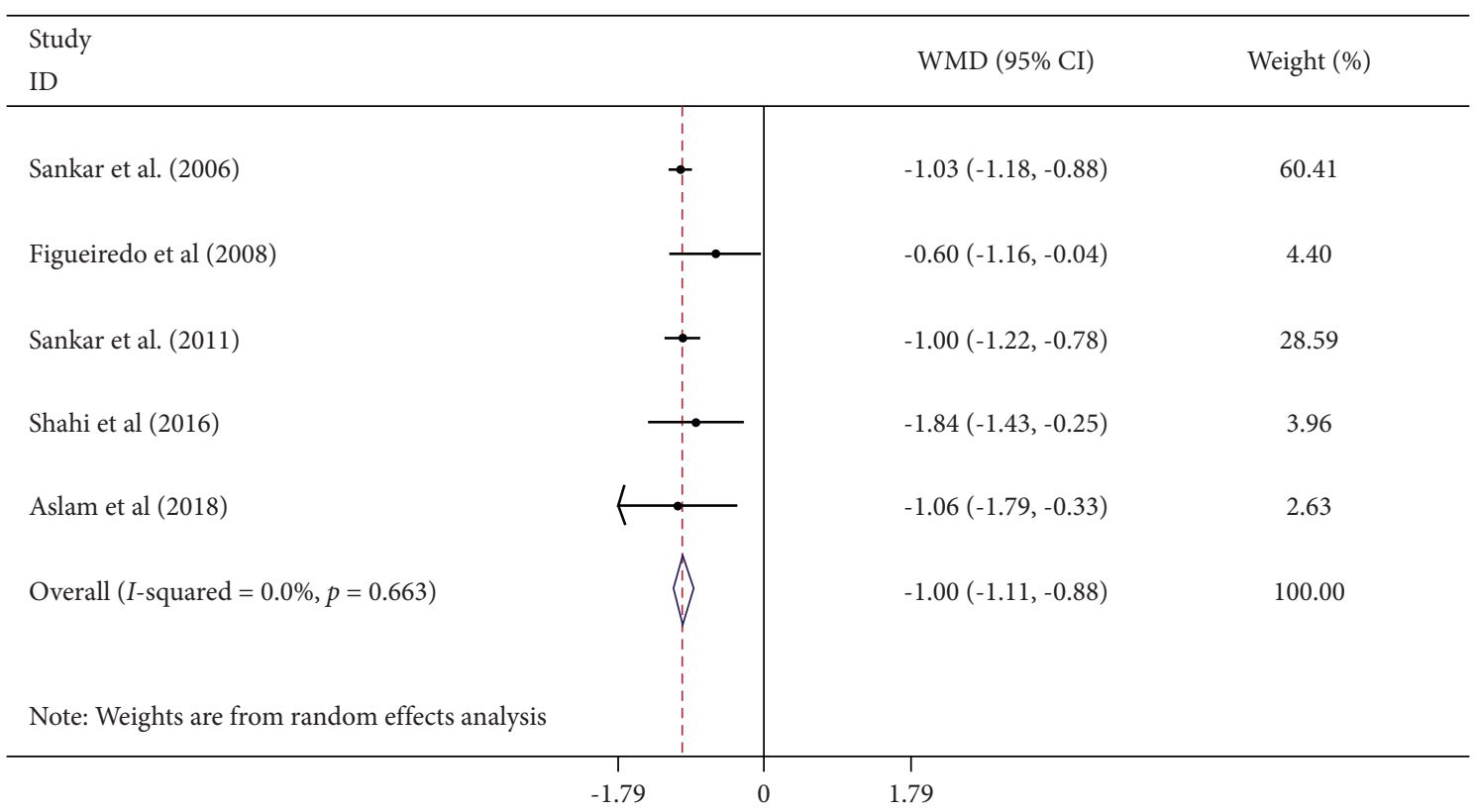

FIGURE 3: Forest plot showing the effects of sesame consumption on circulating HbAlc in adults using the random effects model. Values are WMDs (95\% CIs) comparing changes in HbAlc over time between treatment and control groups. CI, confidence interval; HbA1c, hemoglobin A1c; WMD, weighted mean difference.

TABLE 4: GRADE evidence profile for the effects of sesame supplementation on diabetic indices in adults ${ }^{1}$.

\begin{tabular}{|c|c|c|c|c|c|c|c|c|c|}
\hline \multirow[b]{2}{*}{ Outcome } & \multicolumn{6}{|c|}{ Certainty assessment } & \multirow[b]{2}{*}{ MD (95\% CI) } & \multirow[b]{2}{*}{ Certainty } & \multirow[b]{2}{*}{ Importance } \\
\hline & $\begin{array}{l}\text { No. of } \\
\text { studies }\end{array}$ & Risk of bias & Inconsistency & Indirectness & Impression & $\begin{array}{c}\text { Other } \\
\text { considerations }\end{array}$ & & & \\
\hline $\mathrm{FBS}, \mathrm{mg} / \mathrm{dl}$ & 7 & Serious & Nonserious & Serious & Nonserious & Non & $\begin{array}{r}-28.22 \\
(-39.15 \\
-17.29)\end{array}$ & $\begin{array}{c}\oplus \oplus \bigcirc \bigcirc \\
\text { Low }\end{array}$ & Important \\
\hline $\mathrm{HbA1c}, \%$ & 5 & Serious & Nonserious & Serious & Nonserious & Non & $\begin{array}{c}-1.00(-1.11 \\
-0.88)\end{array}$ & $\begin{array}{c}\oplus \oplus \bigcirc \bigcirc \\
\text { Low }\end{array}$ & Important \\
\hline $\begin{array}{l}\text { Insulin, } \\
\mathrm{IU} / \mathrm{ml}\end{array}$ & 3 & Nonserious & Serious & Serious & Serious & Non & $\begin{array}{c}7.51(-3.81 \\
18.83)\end{array}$ & $\begin{array}{l}\oplus \bigcirc \bigcirc \bigcirc \\
\text { Verylow }\end{array}$ & Important \\
\hline
\end{tabular}

${ }^{1} \mathrm{CI}$, confidence interval; GRADE, Grades of Recommendations, Assessment, Development, and Evaluation; FBS, fasting blood sugar; HbA1c, hemoglobin A1c; MD, mean difference.

\section{Discussion}

This systematic review and meta-analysis study summarizes the findings of eight randomized clinical trials evaluating the effect of sesame consumption on glycemic indices. This study found that sesame consumption has a hypoglycemic effect which decreases serum FBS and HbA1C. The reductions of $28.23 \mathrm{mg} / \mathrm{dl}$ in the FBS level and $1.0 \%$ of HbA1C are clinically significant. A change (either positive or negative) in the A1C percentage of $0.5 \%$ is considered clinically significant [29]. This is important to public health as an increase of $18 \mathrm{mg} / \mathrm{dl}(1 \mathrm{mmol} / \mathrm{l})$ in glucose levels has been associated with a $4 \%$ increase in mortality risk in nondiabetic patients and $5 \%$ in diabetic patients [30]. In this analysis, all of the eligible studies were conducted on patients with type two diabetes. Sesame intake has a larger improvement effect on glycemic indices among diabetic patients due to the high serum level of glycemic markers in this group.
We also found that serum FBS and HbA1C declined among people who consumed sesame. This is consistent with findings from an in vitro study on diabetic rats, which produced evidence that sesamin leads to a significant improvement in the serum FBS and HbAlc in a dose-dependent manner by promoting glycogen synthesis in the liver [12]. Similarly, Devarjan et al. in an open-label randomized dietary intervention study investigated the effects of the combination of sesame oil blend (sesame oil along with rice bran oil) (maximum $35-40 \mathrm{ml} / \mathrm{d}$ as cooking oil) and glibenclamide $(5 \mathrm{mg} / \mathrm{d}$ ) for 8 weeks on glycemic indices in type 2 diabetes mellitus patients as compared to diabetic patients only used glibenclamide. This study also demonstrated the lowering effects of sesame oil blend and glibenclamide on serum FBS and $\mathrm{HbA} 1 \mathrm{C}$ as compared to the glibenclamide group [31]. Furthermore, Mosalaeipour Yazdi et al., in 2008, produced evidence that sesame oil has the ability to improve FBS and HbA1C in type II diabetic 
patients when they carried out a quasiexperimental study lasting 6 weeks assessing the influence of daily intake of $30 \mathrm{~g}$ sesame oil without any control group [32].

These findings differ from a randomized, triple-blind, three-way crossover trial by Raeisi-Dehkordi et al. with 95 subjects who have type 2 diabetes (18-60 years). These patients substituted sesame oil, canola oil, or sesame-canola oil for their regular dietary oil for 9 weeks with a 4 -week washout period. When sesame oil or sesame-canola oil was compared to canola oil, no significant effect on FBS, insulin, and HOMA-IR was observed [26]. However, in this study, participants only replaced dietary oils; there was not a set dosage [26]. This variation in consumption levels may explain the inability to find an effect in this trial [26].

These discrepancies might also be explained by the difference in bioavailability among lignan compounds in sesame products (sesame seeds, sesame lignans, and sesame oil). Most of the studies in this analysis used sesame oil. Sesame oil contains greater quantities of lignan $(11.51 \mathrm{mg} / \mathrm{g})$ [33] than sesame seed $(5.81 \mathrm{mg} / \mathrm{g})$ [34]. Sesame oil also is a rich source of $\alpha$-tocopherol (40 mg/100 g), PUFA (43\%), and MUFA (40\%) [27]. Epidemiological studies have found that substituting foods high in saturated fatty acids with foods high in MUFA and PUFA could be associated with the prevention of diabetes [35]. High amounts of antioxidant compounds (vitamin $\mathrm{E}$ and lignans) in sesame oil may also contribute through powerful radical scavenging effects, protecting B cells from destruction [36].

It is also important to note the lack of consistency regarding placebo substances used for comparison to sesame. These oils (sunflower, canola, soybean, palm, and groundnut oils) contain different levels of PUFA, MUFA, and other biological compounds which can positively influence glycemic markers. It is challenging to quantify the actual effect of sesame on glycemic indices above that of a placebo due to the known therapeutic effect of these oils.

The hypoglycemic effects of sesame may be explained by multiple potential mechanisms. Sesamin can inhibit the augment of blood glucose by playing a role in increasing glycogen production [12]. It has been proposed that sesamin plays a role in glucose uptake, carbohydrate metabolism, and insulin signal transduction pathways by controlling the expression of relevant genes in type 2 diabetic rats [37]. Previous studies have suggested that high levels of dietary MUFA may control glycemic responses by suppressing $\beta$-cell destruction and elevating insulin sensitivity [38, 39]. The mechanism by which sesame decrease HbAlc is unknown, but the antioxidant activity of sesamin may explain some of the actions [16]. Sesame may protect B cells from death by scavenging reactive oxygen species (ROS) [8].

This study has several limitations. The small number of trials identified in the literature suggests that the present systematic review and meta-analysis results may be biased by the sample size. Similarly, eligible studies were found to be heterogeneous regarding sesame preparation, placebo content, duration of intervention, and sample size.

The overall result may have been influenced by the differences in dosing and sourcing of sesame. Subgroup analysis to identify dose-dependence or contrast groups by dosing was not feasible due to variations in the sesame preparations and the difficulty of obtaining accurate dose conversions.

Additionally, several of the studies in this analysis was found to be of poor quality, limiting the generalizability of these findings. Several trials failed to sufficiently describe the process of randomization, allocation concealment, and blinding. There is also a geographic limitation with most of the eligible studies having been conducted in Asian countries. Finally, the majority of trials did not control for the dietary changes of participants during the intervention, which may have influenced the outcome.

The protocol of this study has not been registered in PROSPERO. However, PROSPERO and all relevant databases were comprehensively searched to confirm that no systematic review and meta-analysis has yet been conducted.

\section{Conclusion}

This systematic review produces evidence that sesame consumption could decrease glycemic indices, especially in diabetic patients. This study finds that sesame is a safe and effective complementary approach to improve hyperglycemia in clinical practice. However, the small numbers of related RCTs and high amounts of heterogeneity among these studies limit generalizability. We recommend that additional high-quality RCTs be conducted on participants with different health conditions, varying duration of intervention, and multiple kinds and dosages of sesame consumption to confirm our findings.

\section{Conflicts of Interest}

The authors declare that they have no conflicts of interest.

\section{Authors' Contributions}

Alireza Yargholi and Mohammad Hossein Ayati conceptualized and designed the study and reviewed the protocol for important intellectual content. Alireza Yargholi, Mohammad Hasan Najafi, and Laila Shirbeigi drafted the manuscript and collected data. Mohammad Ali Zareian analyzed the data. It has been edited by Jessie Hawkins as native. All authors read and approved the final manuscript.

\section{Supplementary Materials}

Supplementary Figure 1: analysis of the influence of sesame consumption on serum FBS concentrations in adults. CI, confidence interval; FBS, fasting blood sugar. Supplementary Figure 2: analysis of the influence of sesame consumption on serum $\mathrm{HbA1c}$ concentrations in adults. CI, confidence interval; HbA1c, hemoglobin A1c. Supplementary Figure 3: analysis of the influence of sesame consumption on serum insulin concentrations in adults. CI, confidence interval. Supplementary Figure 4: funnel plot for assessing publication bias in the studies reporting the effects of sesame consumption on serum FBS concentrations in adults. FBS, fasting blood sugar; SE, standard error; WMD, weighted mean difference. Supplementary Figure 5: funnel plot for 
assessing publication bias in the studies reporting the effects of sesame consumption on serum HbAlc concentrations in adults. HbA1c, hemoglobin A1c; SE, standard error; WMD, weighted mean difference. Supplementary Figure 6: funnel plot for assessing publication bias in the studies reporting the effects of sesame consumption on serum insulin concentrations in adults. SE, standard error; WMD, weighted mean difference. (Supplementary Materials)

\section{References}

[1] WH Organization, Global Report on Diabetes, WHO, Geneva, Switzerland, 2016.

[2] M. Naghavi, "Global, regional, and national age-sex specific mortality for 264 causes of death, 1980-2016: a systematic analysis for the Global Burden of Disease Study 2016," Lancet, vol. 390, no. 10100, pp. 1151-1210, 2017.

[3] N. Unwin, N. Unwin, J. Shaw, P. Zimmet, and K. G. M. M. Alberti, "Impaired glucose tolerance and impaired fasting glycaemia: the current status on definition and intervention," Diabetic Medicine, vol. 19, no. 9, pp. 708-723, 2002.

[4] E. P. Paulsen, "Hemoglobin A1c in childhood diabetes," Metabolism, vol. 22, no. 2, pp. 269-271, 1973.

[5] H.-Y. Chang, M. Wallis, and E. Tiralongo, "Use of complementary and alternative medicine among people living with diabetes: literature review," Journal of Advanced Nursing, vol. 58, no. 4, pp. 307-319, 2007.

[6] M. Namiki, "Nutraceutical functions of sesame: a review," Critical Reviews in Food Science and Nutrition, vol. 47, no. 7, pp. 651-673, 2007.

[7] A. Kamal-Eldin, A. Moazzami, and S. Washi, "Sesame seed lignans: potent physiological modulators and possible ingredients in functional foods \& nutraceuticals," Recent Patents on Food, Nutrition \& Agriculture, vol. 3, no. 1, pp. 17-29, 2011.

[8] Q. Jin, Y. Liu, X. Wang, and H. Dai, "Sesamin as a natural antioxidant," Journal of the Chinese Cereals and Oils Association, vol. 20, pp. 89-93, 2005.

[9] T. Phitak, P. Pothacharoen, J. Settakorn, W. Poompimol, B. Caterson, and P. Kongtawelert, "Chondroprotective and anti-inflammatory effects of sesamin," Phytochemistry, vol. 80, pp. 77-88, 2012.

[10] M. Mohammadshahi, M. Zakerzadeh, M. Zakerkish, M. Zarei, and A. Saki, "Effects of sesamin on the glycemic index, lipid profile, and serum Malondialdehyde level of patients with type II diabetes," Journal of Babol University of Medical Sciences, vol. 18, no. 6, pp. 7-14, 2016.

[11] M. Elleuch, S. Besbes, O. Roiseux, C. Blecker, and H. Attia, "Quality characteristics of sesame seeds and by-products," Food Chemistry, vol. 103, no. 2, pp. 641-650, 2007.

[12] L. Hong, W. Yi, C. Liangliang, H. Juncheng, W. Qin, and Z. Xiaoxiang, "Hypoglycaemic and hypolipidaemic activities of sesamin from sesame meal and its ability to ameliorate insulin resistance in KK-Ay mice," Journal of the Science of Food and Agriculture, vol. 93, no. 8, pp. 1833-1838, 2013.

[13] F. Aslam, S. Iqbal, M. Nasir, A. A. Anjum, P. Swan, and K. Sweazea, "Evaluation of white sesame seed oil on glucose control and biomarkers of hepatic, cardiac, and renal functions in male sprague-dawley rats with chemically induced diabetes," Journal of Medicinal Food, vol. 20, no. 5, pp. 448-457, 2017.

[14] M. Mohammad Shahi, M. Zakerzadeh, M. Zakerkish, M. Zarei, and A. Saki, "Effect of sesamin supplementation on glycemic status, inflammatory markers, and adiponectin levels in patients with type 2 diabetes mellitus," Journal of Dietary Supplements, vol. 14, no. 1, pp. 65-75, 2017.

[15] F. Aslam, S. Iqbal, M. Nasir, and A. A. Anjum, "White sesame seed oil mitigates blood glucose level, reduces oxidative stress, and improves biomarkers of hepatic and renal function in participants with type 2 diabetes mellitus," Journal of the American College of Nutrition, vol. 38, no. 3, pp. 235-246, 2019.

[16] D. Sankar, A. Ali, G. Sambandam, and R. Rao, "Sesame oil exhibits synergistic effect with anti-diabetic medication in patients with type 2 diabetes mellitus," Clinical Nutrition, vol. 30, no. 3, pp. 351-358, 2011.

[17] D. Moher, L. Shamseer, L. Shamseer et al., "Preferred reporting items for systematic review and meta-analysis protocols (PRISMA-P) 2015 statement," Systematic Reviews, vol. 4, no. 1, p. 1, 2015.

[18] A. R. Jadad, R. A. Moore, D. Carroll et al., "Assessing the quality of reports of randomized clinical trials: is blinding necessary?" Controlled Clinical Trials, vol. 17, no. 1, pp. 1-12, 1996.

[19] G. W. Group, "Grading quality of evidence and strength of recommendations," BMJ, vol. 328, no. 7454, p. 1490, 2004.

[20] J. Hartung, "An alternative method for meta-analysis," Biometrical Journal, vol. 41, no. 8, pp. 901-916, 1999.

[21] M. Borenstein, L. V. Hedges, J. P. Higgins, and H. R. Rothstein, Introduction to Meta-Analysis, John Wiley \& Sons, Hoboken, NJ, USA, 2011.

[22] J. P. A. Ioannidis, N. A. Patsopoulos, and E. Evangelou, "Uncertainty in heterogeneity estimates in meta-analyses," BMJ, vol. 335, no. 7626, pp. 914-916, 2007.

[23] J. P. Higgins and S. Green, Cochrane Handbook for Systematic Reviews of Interventions, Wiley-Blackwell, Hoboken, NJ, USA, 2008.

[24] A. S. Figueiredo and J. Modesto-Filho, "Effect of defatted sesame (Sesamum indicum L.) flour on the blood glucose level in type 2 diabetic women," Brazilian Journal of Pharmacognosy, vol. 18, no. 1, pp. 77-83, 2008.

[25] M. Golzarand, Z. Bahadoran, S. Hosseinpour-Niazi, S. Mirzaee, F. Azizi, and P. Mirmiran, "Effect of Ardeh on components of metabolic syndrome in type 2 diabetic patients: a randomized clinical trial," Iranian Journal of Endocrinology and Metabolism, vol. 15, no. 4, pp. 333-339, 2013.

[26] H. Raeisi-Dehkordi, M. Amiri, A. Zimorovat et al., "The effects of canola oil compared with sesame and sesame-canola oil on glycemic control and liver function enzymes in patients with type 2 diabetes: a 3-way randomized triple-blind crossover clinical trial," ARYA Atherosclerosis, vol. 15, no. 4, p. e3399, 2019.

[27] D. Sankar, M. R. Rao, G. Sambandam, and K. V. Pugalendi, “A pilot study of open label sesame oil in hypertensive diabetics," Journal of Medicinal Food, vol. 9, no. 3, pp. 408-412, 2006.

[28] A. Farajbakhsh, S. M. Mazloomi, M. Mazidi et al., "Sesame oil and vitamin $\mathrm{E}$ co-administration may improve cardiometabolic risk factors in patients with metabolic syndrome: a randomized clinical trial," European Journal of Clinical Nutrition, vol. 73, no. 10, pp. 1403-1411, 2019.

[29] R. R. Little and C. L. Rohlfing, "The long and winding road to optimal HbA1c measurement," Clinica Chimica Acta, vol. 418, pp. 63-71, 2013.

[30] I. Stranders, M. Diamant, R. E. van Gelder et al., "Admission blood glucose level as risk indicator of death after myocardial infarction in patients with and without diabetes mellitus," 
Archives of Internal Medicine, vol. 164, no. 9, pp. 982-988, 2004.

[31] S. Devarajan, B. Chatterjee, H. Urata et al., "A blend of sesame and rice bran oils lowers hyperglycemia and improves the lipids," The American Journal of Medicine, vol. 129, no. 7, pp. 731-739, 2016.

[32] Y. M. Mosalaeipour, S. Eghtesadi, F. Kaseb, A. M. Afkhami, and F. Hosseini, "Effects of sesame oil on blood glucose and lipid profile in type II diabetic patients referring to the yazd diabetes research center," Journal of Shahid Sadoughi University of Medical Sciences, vol. 16, 2008.

[33] W.-H. Wu, "The contents of lignans in commercial sesame oils of Taiwan and their changes during heating," Food Chemistry, vol. 104, no. 1, pp. 341-344, 2007.

[34] A. A. Moazzami and A. Kamal-Eldin, "Sesame seed is a rich source of dietary lignans," Journal of the American Oil Chemists Society, vol. 83, no. 8, p. 719, 2006.

[35] U. Risérus, W. Willett, and F. Hu, "Dietary fats and prevention of type 2 diabetes," Progress in Lipid Research, vol. 48, no. 1, pp. 44-51, 2009.

[36] M. Nakai, M. Harada, K. Nakahara et al., "Novel antioxidative metabolites in rat liver with ingested sesamin," Journal of Agricultural and Food Chemistry, vol. 51, no. 6, pp. 1666-1670, 2003.

[37] P. Bigoniya, R. Nishad, and C. S. Singh, "Preventive effect of sesame seed cake on hyperglycemia and obesity against high fructose-diet induced type 2 diabetes in rats," Food Chemistry, vol. 133, no. 4, pp. 1355-1361, 2012.

[38] B. J. Brehm, B. L. Lattin, S. S. Summer et al., "One-year comparison of a high-monounsaturated fat diet with a highcarbohydrate diet in type 2 diabetes," Diabetes Care, vol. 32, no. 2, pp. 215-220, 2009.

[39] F. Haidari, M. Mohammadshahi, M. Zarei, and Z. Gorji, "Effects of sesame butter (Ardeh) versus sesame oil on metabolic and oxidative stress markers in streptozotocininduced diabetic rats," Iranian Journal of Medical Sciences, vol. 41, no. 2, pp. 102-109, 2016. 